\title{
Mechanochemistry on the Müller-Brown Surface by Newton Trajectories
}

\author{
Wolfgang Quapp*, Josep Maria Bofill ${ }^{\dagger}$
}

September 23, 2017

\begin{abstract}
Chemical processes which suffer the application of mechanical force are theoretically described by effective potential energy surfaces (PES). We worked out [Theor. Chem. Acc. 135, 113 (2016)] that the changes due to the force for the minimums and for the saddle points can be described by Newton trajectories (NT) of the original PES. If the force is so high that the saddle point disappears into a shoulder then the mechanochemical action is fulfilled: the pulling force breaks down the reaction barrier. The point is named barrier breakdown point (BBP). Different families of NTs form corridors on the original PES which describe qualitative different actions of the force. The border regions of such corridors are governed by the valley-ridge inflection points (VRI) of the surface. Here we discuss all this on the basis of the well known Müller-Brown (MB) surface, and we describe a new kind of NT-corridor.

Keywords: Mechanochemistry, Effective PES, Newton trajectory, Force displaced stationary points
\end{abstract}

\section{Introduction to Newton Trajectory Theory applied to Mechanochemistry}

Mechanochemistry is an emergent area based on the study of forces that initialize chemical reactions. A mechanical force applied to a material is not only used for crushing solids but it can also be used for the initiation of chemical reactions. ${ }^{1-3}$ From a theoretical point of view, the potential energy surface (PES) of the molecule changes under the force, which means that the minimums and the saddle points (SPs), thus the barriers between the minimums and the SPs, change. It means that also the reaction pathways of the chemical problem change. For this reason, when a force is applied to a molecular system, the reactivity is either enhanced or suppressed. The extent of the barrier modification depends on the direction and on the magnitude of the external force.

\footnotetext{
*Mathematisches Institut, Universität Leipzig, PF 100920, D-04009 Leipzig, Germany, quapp@uni-leipzig.de

†Departament de Química Orgànica, Universitat de Barcelona, and Institut de Química Teòrica i Computacional, Universitat de Barcelona, (IQTCUB), Martí i Franquès, 1, 08028 Barcelona, Spain, jmbofill@ub.edu
} 


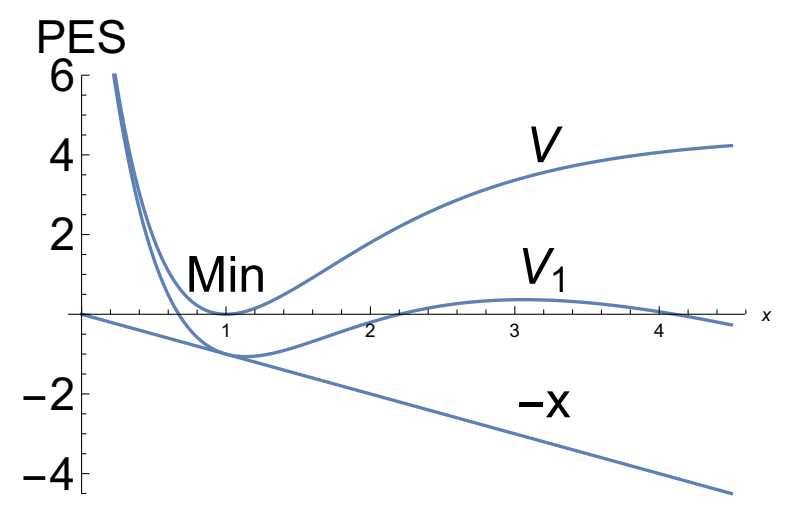

Figure 1: An 1-dimensional Morse potential, V, is the upper curve. The effective surface under the amount of force, -1 , emerges as $V_{1}$. Note that minimum and $\mathrm{SP}$ of $\mathrm{V}_{1}$ are moved.

In Fig.1 the simplest 1-dimensional case is shown: to a potential $V(x)$ is added an external constant force of amount, -1 . We get

$$
V_{1}(x)=V(x)-1 \cdot x .
$$

The force induces that minimum and SP of the original potential move; here the minimum moves to the right hand side, but the SP moves to the left hand side. If the amount of the force, F, is larger, then the line in Fig. 1 becomes steeper, and the movement of the stationary points increases.

What happens on a 2-dimensional PES? Of course, we can apply Eq.(1) to every curve section for a fixed $y$ value, over the then 1 -dimensional curve over an $x$-axis. Equivalently, we can apply

$$
V_{F}(x, y)=V(x, y)-F \cdot y
$$

in $y$-direction, for every fixed value of $x$. The corresponding 1-dimensional curve over an $y$-axis is changed like in Fig.1. In the sum, for a two-dimensional surface, we can combine the two kinds of external forces to one directional vector, $\mathbf{f}=\left(\mathrm{F}_{x}, \mathrm{~F}_{y}\right)^{T}$, and we get the model

$$
V_{\mathbf{f}}(x, y)=V(x, y)-F_{x} \cdot x-F_{y} \cdot y=V(x, y)-\left(F_{x}, F_{y}\right) \cdot(x, y)^{T} .
$$

The last point symbol, ' ', now means the scalar product of the vectors, $\mathbf{f}$ and (x,y), and the superscript $T$ means the transposition of a vector or a matrix. The amount of the force in direction $\mathbf{f}$ is the norm of the vector, $\sqrt{F_{x}^{2}+F_{y}^{2}}$. If the potential function $V(x, y)$ is approximated by a quadratic model then we have the Thronton model ${ }^{4}$ used to predict the resulting structure and location of the transition state due to changes on the original system.

In the general case, we collect all coordinates in a coordinate vector, $\mathbf{x}$, and the $N$ dimensional force in the vector, $\mathbf{f}$. The resulting modified PES, $V_{\mathbf{f}}(\mathbf{x})$, is obtained for a constant stretching force, $\mathbf{f}$, via $^{3,5-12}$

$$
V_{\mathbf{f}}(\mathbf{x})=V(\mathbf{x})-\mathbf{f}^{T} \cdot\left(\mathbf{x}-\mathbf{x}_{0}\right)
$$

where $\mathbf{x}$ is the vector of the internal coordinates of the molecule, and $\mathbf{x}_{0}$ is any anchor point. The vector of the mechanical force, $\mathbf{f}$, is determined by two parts: its direction and its length. 
The 'length' is the norm of the vector: $F= \pm|\mathbf{f}|$, and the direction is $\mathbf{l}=\mathbf{f} / F$. So, $\mathbf{l}$ is a unit vector. According to Eq.(4) the effective potential results by a subtraction of the scalar product of a linear potential, the displacement, $\left(\mathbf{x}-\mathbf{x}_{0}\right)$, with the force vector, $\mathbf{f}$, from the original potential energy, $V(\mathbf{x})$.

There is a slightly other ansatz, ${ }^{13}$ in comparison to Eq.(4), the force-modified potential energy surface. It is an extension of a Kauzmann-Eyring model, whith an expression $V_{f}(\mathbf{r})$ $=V(\mathbf{r})+\mathrm{f}_{0}\left\|\overline{\mathbf{r}}_{0}-\overline{\mathbf{r}}\right\|$. Here $f_{0}$ is the magnitude of the external force, but not the force vector. The gradient formally is $\nabla_{\mathbf{r}} V_{f}(\mathbf{r})=\nabla_{\mathbf{r}} V(\mathbf{r})-f_{0}\left(\mathbf{r}_{0}-\mathbf{r}\right)\left\|\mathbf{r}_{0}-\mathbf{r}\right\|^{-1}$. However, for $\overline{\mathbf{r}}$ in the external force are used only special molecular attachment points, and the direction of the force depends on this special coordinate direction, $\overline{\mathbf{r}}$. It is not constant. But some phenomenons there observed are similar to our results. See also a longer comparison elsewhere. ${ }^{14}$

The Maeda-group ${ }^{15-17}$ uses an application of another non-linear ansatz of a force vector, instead of Eq.(4), the Artificial Force Induced Reaction (AFIR) method. However, the version is used only for numerical reasons to calculate transition states or minimums. It is described as a method without a physical meaning. Some phenomenons there observed are similar to our results.

\subsection{The invariance of Eq.(4) under coordinate transformation.}

Dealing with the invariance of Eq.(4) we take into account that the transformed potential function, $V_{\mathbf{f}}(\mathbf{x})$, can be considered as the Legendre transformation of $V(\mathbf{x}),{ }^{11}$ where, $\mathbf{f}=$ $\nabla_{\mathbf{x}} V(\mathbf{x})$ and without loss of generality we take $\mathbf{x}_{0}=\mathbf{0}$. Let first $\mathbf{x}=\mathbf{r}(\mathbf{q})$ be a regular transformation of the coordinates $\mathbf{x}$ alone into new coordinates $\mathbf{q}$. The following question emerges: is it possible to find a vector function, $\mathbf{s}(\mathbf{q}, \mathbf{p})$, such that the transformation

$$
\begin{aligned}
& \mathbf{x}=\mathbf{r}(\mathbf{q}) \\
& \mathbf{f}=\mathbf{s}(\mathbf{q}, \mathbf{p}),
\end{aligned}
$$

transforms Eq.(4) into a new Legendre transformed function $U_{\mathbf{p}}(\mathbf{q})=U(\mathbf{q})-\mathbf{p}^{T} \mathbf{q}$ ? We require that the Jacobian

$$
\operatorname{det}\left(\begin{array}{cc}
\nabla_{\mathbf{q}} \mathbf{r}^{T} & \nabla_{\mathbf{q}} \mathbf{s}^{T} \\
\nabla_{\mathbf{p}} \mathbf{r}^{T} & \nabla_{\mathbf{p}} \mathbf{s}^{T}
\end{array}\right)=\operatorname{det}\left(\begin{array}{cc}
\nabla_{\mathbf{q}} \mathbf{r}^{T} & \nabla_{\mathbf{q}} \mathbf{s}^{T} \\
\mathbf{O} & \nabla_{\mathbf{p}} \mathbf{s}^{T}
\end{array}\right)=\operatorname{det}\left(\nabla_{\mathbf{q}} \mathbf{r}^{T}\right) \operatorname{det}\left(\nabla_{\mathbf{p}} \mathbf{s}^{T}\right)
$$

is not zero. The answer is affirmative by the following simple argument. The original potential function $V(\mathbf{x})$ is equal to the potential function $U(\mathbf{q})$ via

$$
V(\mathbf{x})=V(\mathbf{r}(\mathbf{q}))=U(\mathbf{q})
$$

Hence if we introduce

$$
\mathbf{p}=\nabla_{\mathbf{q}} U(\mathbf{q})
$$

then the corresponding transformed function

$$
U_{\mathbf{p}}(\mathbf{q})=U(\mathbf{q})-\mathbf{p}^{T} \mathbf{q}
$$


is obtained as transformed Legendre function. In order to compute the vector function, $\mathbf{s}(\mathbf{q}, \mathbf{p})$ (in Eq. $(5 \mathrm{~b}))$, we compute

$$
\mathbf{p}=\nabla_{\mathbf{q}} U(\mathbf{q})=\left[\nabla_{\mathbf{q}} \mathbf{r}^{T}\right] \nabla_{\mathbf{x}} V(\mathbf{x})=\left[\nabla_{\mathbf{q}} \mathbf{r}^{T}\right] \mathbf{f}
$$

where Eqs. (5a) and (7) have been used. We assume $\operatorname{det}\left(\nabla_{\mathbf{q}} \mathbf{r}^{T}\right) \neq 0$. Therefore one can solve Eq.(10) for $\mathbf{f}$ and one obtains $\mathbf{f}=\mathbf{s}(\mathbf{q}, \mathbf{p})$ even as a linear function in $\mathbf{p}$. One easily checks that $\operatorname{det}\left(\nabla_{\mathbf{p}} \mathbf{s}^{T}\right) \neq 0$. Finally, if we consider the invariance of the scalar product, $\mathbf{f}^{T} \mathbf{x}=\mathbf{p}^{T} \mathbf{q}$, under the transformation included in Eqs.(5) satisfying the condition of Eq.(6) and of Eq.(7), we conclude that $V_{\mathbf{f}}(\mathbf{x})=U_{\mathbf{p}}(\mathbf{q})$.

Note that the invariance of Eq.(4) corresponds to the known invariance of Newton trajectories (NTs) under coordinate transformation, ${ }^{18}$ which becomes clear below because NTs are connected with Eq.(4).

\subsection{The Mechanochemical Model}

Eq.(4) is the basis of some mechanochemical methods for the calculation of deformed molecular structures due to a force. It can also be applied to explore how an external electric fields can catalyze and control a reaction when the field is constant in time and position. ${ }^{19}$ The dimension of the vectors, $\mathbf{f}, \mathbf{x}$, and $\mathbf{x}_{0}$ is $N=3 n-6$ internal coordinates being $n$ the number of atoms. On the effective potential, $V_{\mathbf{f}}(\mathbf{x})$, the stationary points are located at different points with respect to the unperturbed potential, $\mathrm{V}(\mathbf{x})$, where $\nabla_{\mathbf{x}} V(\mathbf{x})=\mathbf{g}(\mathbf{x})=\mathbf{0}$ holds. The stationary points on the effective potential have to satisfy the analogous condition, $\nabla_{\mathbf{x}} V_{\mathbf{f}}(\mathbf{x})=\mathbf{0}$. Since $V_{\mathbf{f}}(\mathbf{x})$ is the one given in Eq.(4) it follows that its minimums and SPs should satisfy the vector equation

$$
\nabla_{\mathbf{x}} V_{\mathbf{f}}(\mathbf{x})=\mathbf{g}(\mathbf{x})-\mathbf{f}=\mathbf{0}
$$

One searches a point where the gradient of the original PES, $\mathbf{g}(\mathbf{x})$, has to be equal to the mechanochemical force, f. From a geometrical point of view, Eq.(11) means that the tangential hyperplane at a point $\mathbf{x}$ of the original PES, characterized by the gradient, $\mathbf{g}(\mathbf{x})$, is equal to the hyperplane made by the pulling force, by $\mathbf{f}^{T} \cdot\left(\mathbf{x}-\mathbf{x}_{0}\right)$, of Eq.(4).

If it is $F=0$ then Eq.(4) reduces to the pure 'thermal limit' being the case without a mechanical load.

Let us consider the case that the force $\mathbf{f}$ changes its magnitude $F$ in a continuous way but its direction $\mathbf{l}$ is constant. Thus along the curve of solutions of Eq.(11) the gradient points into the same direction where its length will change. In this case we have a continuous set of effective PESs, $V_{\mathbf{f}}(\mathbf{x})$, where every $V_{\mathbf{f}}(\mathbf{x})$ has its corresponding stationary points. However, if $F$ changes then this set of stationary points defines a continuous curve. We can draw the curve over the original PES, $V(\mathbf{x})$. By this construction, at each point of the curve the equation, $\pm F \mathbf{l}=\mathbf{g}(\mathbf{x})$, holds, see Eq.(11).

We summarize: at each point of the pathway of the moved stationary points the gradient has the same direction, 1. A curve with this feature is known as Newton trajectory (NT), ${ }^{20-27}$ or more specifically here as the curve of the force displaced stationary points (FDSPs). ${ }^{28,29}$ 


\section{Application of Newton Trajectories}

It is known that every NT satisfies the Branin equation ${ }^{19,28,30,31}$

$$
\frac{d \mathbf{x}}{d s}= \pm \mathbf{A}(\mathbf{x}) \mathbf{g}(\mathbf{x})
$$

where $s$ is the parameter that characterizes the curve, and the matrix $\mathbf{A}(\mathbf{x})$ is computed as a product of the determinant of the Hessian matrix, $\mathbf{H}(\mathbf{x})$, of the original PES, $V(\mathbf{x})$, with its inverse, $\mathbf{A}(\mathbf{x})=\operatorname{Det}(\mathbf{H}(\mathbf{x}))(\mathbf{H}(\mathbf{x}))^{-1}$. The matrix $\mathbf{A}(\mathbf{x})$ is named the adjoin matrix of the Hessian, $\mathbf{H}(\mathbf{x})$. Eq.(12) is also a way to generate the FDSPs curve.

Eq.(11) can be written in a projector form ${ }^{20,21}$

$$
\left(\mathrm{U}-1 \mathbf{l}^{T}\right) \mathrm{g}=\mathbf{0}
$$

where $\mathbf{U}$ is the unit matrix. The equation has to hold unattached from the uncomfortable norm, $|\mathbf{g}|$, and it means nothing else that $\mathbf{g}$ and $\mathbf{l}$ are parallel. If we differentiate the projector Eq.(13) with respect to $s$, we obtain ${ }^{21,24}$

$$
\left(\mathbf{U}-\mathbf{l} \mathbf{l}^{T}\right) \mathbf{H} \frac{d \mathbf{x}}{d s}=\mathbf{0} .
$$

This is a second expression of the tangent of the FDSPs curve.

If one uses a given fixed direction, $\mathbf{l}$, and if one goes along the corresponding FDSPs curve then the magnitude of the force, $F$, starts with zero at a stationary point, and ends with zero at the final next stationary point. In between there has to be a maximum of $|\mathbf{g}|$. Here holds the condition ${ }^{3,28,29}$

$$
\operatorname{Det}(\mathbf{H}(\mathbf{x}))=0
$$

with the Hessian of the original PES, $V(\mathbf{x})$. The latter case is the point where the square of the gradient norm achieves a turning point, and the effective $V_{\mathbf{f}}(\mathbf{x})$ along the FDSPs path here achieves a shoulder. ${ }^{3,28,29}$ The point on the FDSPs curve has been named barrier breakdown point (BBP). ${ }^{28,29}$ The barrier of $V_{\mathbf{f}}(\mathbf{x})$ decreases from the original PES barrier to zero at the BBP. The kind of points is discussed also elsewhere. ${ }^{14}$

\subsection{A general proof of Eq.(15) for BBPs: ${ }^{28}$}

To prepare the idea of the proof of condition Eq.(15) we remark that the BBP is a turning point of the function $|\mathbf{g}|$ along the NT. $\mathbf{A} \mathbf{g}=\left(\mathbf{g}^{T} \mathbf{A}\right)^{T}=\mathbf{A}^{T} \mathbf{g}$ is the tangent direction of the NT, see Eq.(12), because the matrix $\mathbf{A}$ is symmetric. We search the turning point (TP) of the function $|\mathbf{g}|$ along the NT: the directional derivative of $|\mathbf{g}|$ along the NT has to be zero.

$$
\frac{d}{d s} \sqrt{\mathbf{g}^{T} \mathbf{g}}=\frac{2}{\sqrt{\mathbf{g}^{T} \mathbf{g}}} \mathbf{g}^{T} \mathbf{H} \frac{d \mathbf{x}}{d s}= \pm \frac{2}{\sqrt{\mathbf{g}^{T} \mathbf{g}}} \mathbf{g}^{T} \mathbf{H A g}= \pm 2 \sqrt{\mathbf{g}^{T} \mathbf{g}} \operatorname{det}(\mathbf{H})=0
$$

where first the concept of directional derivative is used and second the tangent vector, $d \mathbf{x} / d s$, is replaced by the definition of Branin, Eq.(12). The proof is finished: it is easy because we have the tool of NTs at hand. 


\subsection{The manifold of BBPs}

The BBPs define a manifold, or a curve in the two-dimensional case. A way to generate a curve with condition (15) is a predictor-corrector method with predictor steps along the tangent, $\mathbf{t}$, by solution of

$$
\frac{d}{d t} \operatorname{Det}(\mathbf{H}(\mathbf{x}(t)))=\left[\nabla_{\mathbf{x}} \operatorname{Det}(\mathbf{H}(\mathbf{x}(t)))\right]^{T} \mathbf{t}=0
$$

The expression $d / d t=\mathbf{t} \boldsymbol{\Delta}_{x}$ is the directional derivative along the tangent vector, $\mathbf{t}$, with $\mathbf{t}=d \mathbf{x} / d t$. The second equality of Eq.(17) is a homogeneous system of $N$ linear equations. The derivation in formula (17) is uncomfortable for larger values of $N$. We consider the usual case that the Hessian matrix has only one zero eigenvalue. The contrary case is seldom and is here excluded. On the MB surface, only near the point $(-1,-0.05)$ we find a crossing of two lines with such a case. We can use the Jacobi formula ${ }^{32}$

$$
\frac{d}{d t} \operatorname{Det}(\mathbf{H}(\mathbf{x}(t)))=\text { Trace }\left[\mathbf{A}(\mathbf{x}(t)) \frac{d}{d t} \mathbf{H}(\mathbf{x}(t))\right] .
$$

As above, the matrix $\mathbf{A}(\mathbf{x})$ is the adjoin matrix of the Hessian, $\mathbf{H}(\mathbf{x})$. For the both matrices is valid: they have the same eigenvectors, and for the eigenvalues $\lambda_{i}$ of $\mathbf{H}$ and $\mu_{j}$ of $\mathbf{A}$ we have

$$
\lambda_{i} \mu_{i}=\operatorname{Det}(\mathbf{H}), \quad i=1, \ldots, N
$$

thus

$$
\mu_{i}=\prod_{\substack{j=1 \\ j \neq i}}^{N} \lambda_{j}
$$

If only one $\lambda_{i}$ is zero, then $\mu_{i} \neq 0$; but all other $\mu_{k}=0$ for $k \neq i$. Let be $\mathbf{E}$ the matrix collecting the set of normalized eigenvectors of the $\mathbf{A}$ matrix, which are also the eigenvectors of $\mathbf{H}$. If we use the properties of Trace we can write

$$
\operatorname{Trace}\left[\mathbf{E} \mu \mathbf{E}^{T} \frac{d \mathbf{H}}{d t}\right]=\operatorname{Trace}\left[\mu \mathbf{E}^{T} \frac{d \mathbf{H}}{d t} \mathbf{E}\right]=\mu_{i} \mathbf{e}_{i}^{T} \frac{d \mathbf{H}}{d t} \mathbf{e}_{i} .
$$

$\mu$ is the diagonal matrix of the eigenvalues of $\mathbf{A}$. Since $\mu_{i} \neq 0$ then must be $\mathbf{e}_{i}^{T} \frac{d \mathbf{H}}{d t} \mathbf{e}_{i}=0$. Now we use the directional derivative along the tangent vector $\mathbf{t}$ and we get

$$
\mathbf{e}_{i}^{T} \frac{d \mathbf{H}}{d t} \mathbf{e}_{i}=\sum_{k=1}^{N} \sum_{l=1}^{N} \sum_{m=1}^{N} e_{i k} \frac{\partial^{3} V}{\partial x_{k} \partial x_{l} \partial x_{m}} e_{l i} \frac{d x_{m}}{d t}=\mathbf{e}_{i}^{T}\langle\mathbf{F}(\mathbf{x})\rangle_{i} \mathbf{t}=\mathbf{w}_{i}^{T} \mathbf{t}=0
$$

$e_{i k}$ is the component $k$ of the vector $\mathbf{e}_{i}$, and $\langle\mathbf{F}(\mathbf{x})\rangle_{i}$ is the matrix of the contraction of the third derivative tensor with the eigenvector $\mathbf{e}_{i}$. It is used to build the vector $\mathbf{w}_{i}=\langle\mathbf{F}(\mathbf{x})\rangle_{i} \mathbf{e}_{i}$. Thus in our normal case of one zero eigenvalue of the Hessian, $\mathbf{t}$ is the orthogonal vector to $\mathbf{w}_{i}$. Comparing the right-hand side part of Eqs. (17) and (22) we see that $\mathbf{w}_{i}=\nabla_{\mathbf{x}} \operatorname{Det}(\mathbf{H}(\mathbf{x}(t)))$. If we have the $\mathbf{w}_{i}$ vector then we can compute the vector $\mathbf{t}$ by the projector

$$
\mathbf{t}=c\left(\mathbf{U}-\frac{\mathbf{w}_{i} \mathbf{w}_{i}^{T}}{\mathbf{w}_{i}^{T} \mathbf{w}_{i}}\right) \mathbf{z}
$$


with an arbitrary vector $\mathbf{z} \neq \mathbf{0}$, and a normalization factor $c$. The matrix $\mathbf{U}$ is the unit matrix of dimension $N \times N$. The calculation is not so expensive as an iteration of the Gauss-Newton algorithm to find optimal BBPs. ${ }^{33}$ Eq. (22) tells us that following the direction of the $\mathbf{w}_{i^{-}}$ vector the unique eigenvector $\mathbf{e}_{i}$ with null eigenvalue of the Hessian matrix, $\mathbf{H}(\mathbf{x})$, loses the nullity character, but along an orthogonal displacement until first order the nullity character is preserved. Note that there is an infinite number of directions orthogonal to the $\mathbf{w}_{i}$-vector. The general equations corresponding to the case of a subset of null eigenvalues of the Hessian matrix will be shown elsewhere.

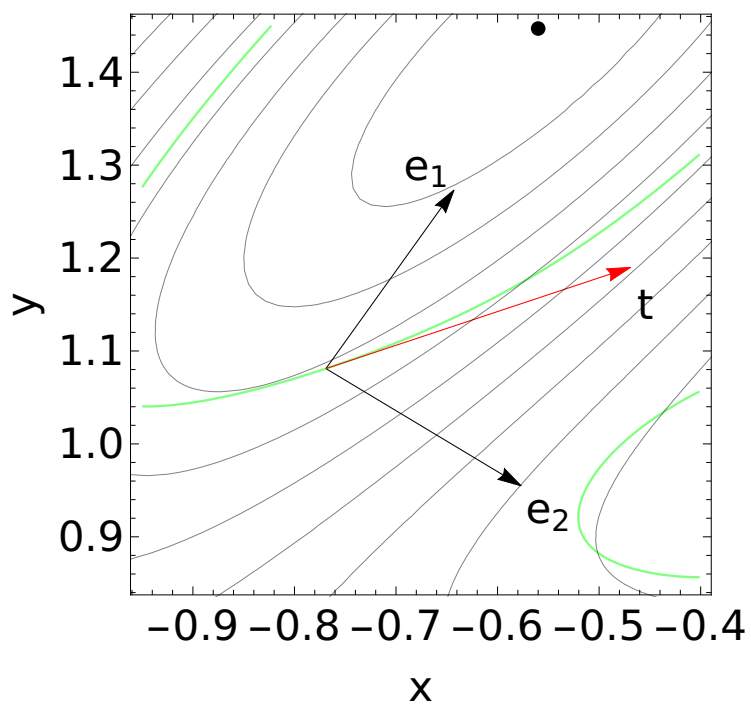

Figure 2: MB surface by Eq.(25). In red color is shown a tangent vector to the line of the green solution curve to $\operatorname{Det}(\mathbf{H}(\mathbf{x}(t)))=0$. $\mathbf{e}_{1}$ and $\mathbf{e}_{2}$ are the eigenvectors of the Hessian at the curve point of interest. $\mathbf{e}_{1}$ belongs to the zero eigenvalue.

In Fig. 2 we show a tangent to a green line with $\operatorname{Det}(\mathbf{H}(\mathbf{x}(t)))=0$ in the main valley on the MB surface. It is calculated by Eq.(23). Shown are also the two eigenvectors. $\mathbf{e}_{1}$ is the eigenvector to the zero eigenvalue.

The first $\operatorname{Det}(\mathbf{H})=0$-manifold that each NT crosses gives the BBP of this NT. If we compare all NTs of a set which connect the same minimum and SP, then the NT which gives the lowest value of $F_{\max }$ is named the optimal $N T$ and the point of intersection with the $\operatorname{Det}(\mathbf{H})=0$-manifold optimal $B B P{ }^{28,29}$ The gradient of the original PES at a BBP gives the direction of the force and its magnitude to enforce the reaction task. The optimal BBP defines the lowest maximal force in magnitude and in a corresponding direction to be applied, in comparison to the FDSPs curves of other directions. The optimal BBP satisfies the vector equation ${ }^{28,29}$

$$
\mathbf{H}(\mathbf{x}) \mathbf{g}(\mathbf{x})=\mathbf{0}
$$

where $\mathbf{g}(\mathbf{x}) \neq 0$. Thus at the optimal BBP the gradient is an eigenvector of the Hessian matrix with null eigenvalue. Such a point is on a gradient extremal (GE), ${ }^{34-40}$ and that is the proof of Eq.(24): the GE coincides at least in a point with the $\operatorname{Det}(\mathbf{H})=0$-manifold. In this special BBP, the $\operatorname{Det}(\mathbf{H})=0$-manifold, the GE and the optimal regular NT meet. The optimal BBP is a stationary point on the function $|\mathbf{g}|$ through the actual equipotential 
curve crossed by the optimal NT. (Note: the GE and the NT are one-dimensional curves in all cases, here in a two-dimensional PESs is the condition $\operatorname{Det}(\mathbf{H})=0$ a line, but it is a manifold in higher dimensions.)

Corollary If during the integration of a curve wholly located in the manifold $\operatorname{Det}(\mathbf{H}(\mathbf{x}))=0$ the condition, $\mathbf{e}_{i}^{T} \mathbf{g}(\mathbf{x}) /|\mathbf{g}(\mathbf{x})|=1$, is satisfied then this point is an optimal BBP. In the contrary case, if $\mathbf{e}_{i}^{T} \mathbf{g}(\mathbf{x})=0$, then the point is a VRI point. In the last case, the analysis of the diagonalized matrix $\langle\mathbf{F}(\mathbf{x})\rangle_{i}$ gives the topography of the VRI point.

From a mathematical point of view the BBP concept is strongly related to the Catastrophe Theory. ${ }^{41-44}$ For this theory, a BBP represents a catastrophe of the changing PES function, $V(\mathbf{x})$, being unfolded by a force affected through the additional perturbation term, $\mathbf{f}^{T} \cdot\left(\mathbf{x}-\mathbf{x}_{0}\right)$, of Eq.(4). Usually an SP of index one and a minimum coalesce to a shoulder, and for still higher forces the two former stationary points totally disappear. This is the meaning of the word Catastrophe: a qualitative change of the effective PES.

The application part of this paper is organized as follows: The next section 3 gives a review on NTs and BBPs on the Müller-Brown (MB) surface. ${ }^{45}$ Section 4 develops the corridors on the MB surface with NTs of a qualitative equal behavior, whereas in Section 5 our goal is to explain the change of different effective surfaces in the diverse corridors. Note that similar treatments for other test surfaces are given elsewhere, ${ }^{19,28,29}$ however here a new effect emerges due to the strong nonlinearity of the MB surface, and the fact that an SP of index two is missed. The last section 6 presents a list of conclusions of this study.

\section{Singular NT, and optimal BBP on the MB surface}

A well-known test function in Theoretical Chemistry is the MB function. ${ }^{45}$ Over a twodimension plane it is with

$$
\begin{gathered}
A=(-200,-100,-170,15), a=(-1,-1,-6.5,0.7), b=(0,0,11,0.6), \\
c=(-10,-10,-6.5,0.7), x_{0}=(1,0,-0.5,-1), y_{0}=(0,0.5,1.5,1) \\
V(x, y)=\sum_{i=1}^{4} A(i) \operatorname{Exp}\left[a(i)\left(x-x_{0}(i)\right)^{2}+b(i)\left(x-x_{0}(i)\right)\left(y-y_{0}(i)\right)+c(i)\left(y-y_{0}(i)\right)^{2}\right] .
\end{gathered}
$$

The contours of the function are shown in Fig. 3. The global minimum is inside a long, narrow, but flat valley being located near the point $(-0.5,1.5)$. It may be the reactant, $R$, of a chemical problem. Further there exists an intermediate minimum, $I$, near $(0,0.45)$ and a product minimum, $P$, near $(0.65,0)$.

The valley-ridge inflection points (VRI) of the surface define a special sort of NTs, the singular NTs. A regular NT directly leads from a minimum to an SP of index one (index theorem $\left.{ }^{47}\right)$. A singular NT bifurcates at the respective VRI point. It can connect two

minimums, or the two bifurcating branches can connect two neighboring SPs of index one, or the two bifurcating branches can connect a minimum with an SP of index two. The last 

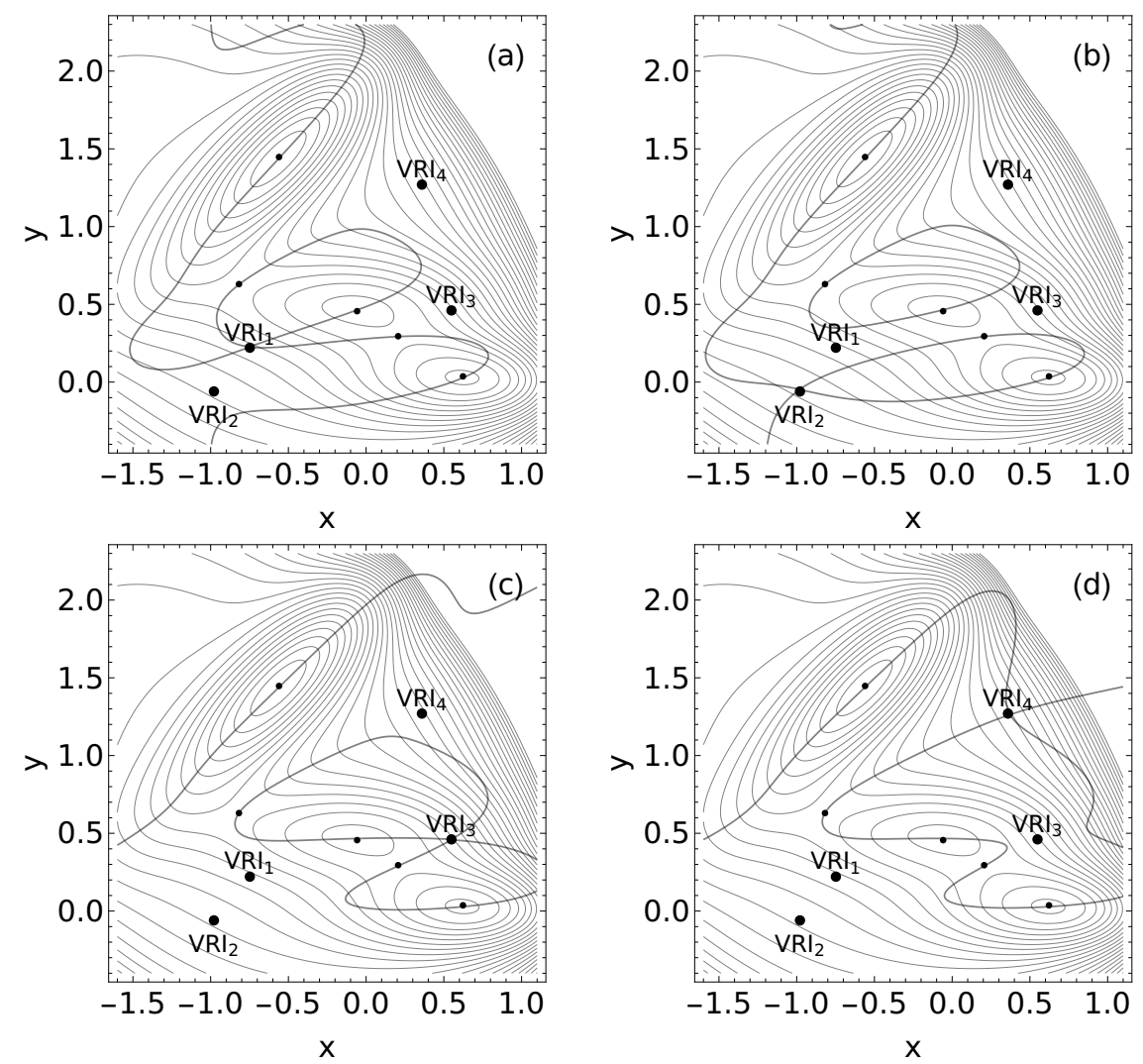

Figure 3: Singular NTs through the four VRIs of the MB surface. The VRI points are marked by black bullets.

case is missing on the MB surface. The singular NTs form the borders of families of regular NTs in between. Such families are named chemical corridors for a pulling. ${ }^{19,28,29}$

In Fig. 3 we show the singular NTs which cross the VRI of the MB surface. ${ }^{47}$ The panels (a) to (d) show the singular NTs through the VRI points 1 to 4 . Note that the singular NTs through $\mathrm{VRI}_{1}$ and through $\mathrm{VRI}_{4}$ connect all stationary points of the MB surface. However, different branches of the singular NTs through $\mathrm{VRI}_{2}$ and through $\mathrm{VRI}_{3}$ only connect a subset of the stationary points of the MB surface. The NTs are disconnected if one takes into account all stationary points. All singular NTs form closed loops of some of their branches, and the singular $\mathrm{NT}$ through $\mathrm{VRI}_{2}$ forms a single, disconnected loop through $\mathrm{SP}_{1}$ and the intermediate minimum. The reason is that here an SP of index 2 does not exists. We will see the consequences of such a disconnection in the next Section 4.

Between different singular NTs, which play the role of borders, we get families of regular NTs. These form the chemical corridors for a pulling, see later Section 4. The importance of the optimal BBP in the theory of Mechanochemistry resides in the fact that it gives the optimal direction and magnitude of the pulling force inside a given corridor. An algorithm to find this type of points is recently discussed elsewhere. ${ }^{33}$

The manifold of points ${ }^{12,28,44}$ where $\operatorname{Det}(\mathbf{H}(x, y))=0$ is characterized by the green lines in Fig.4. The optimal BBPs between the stationary points are the fat, red points in Fig.4. 


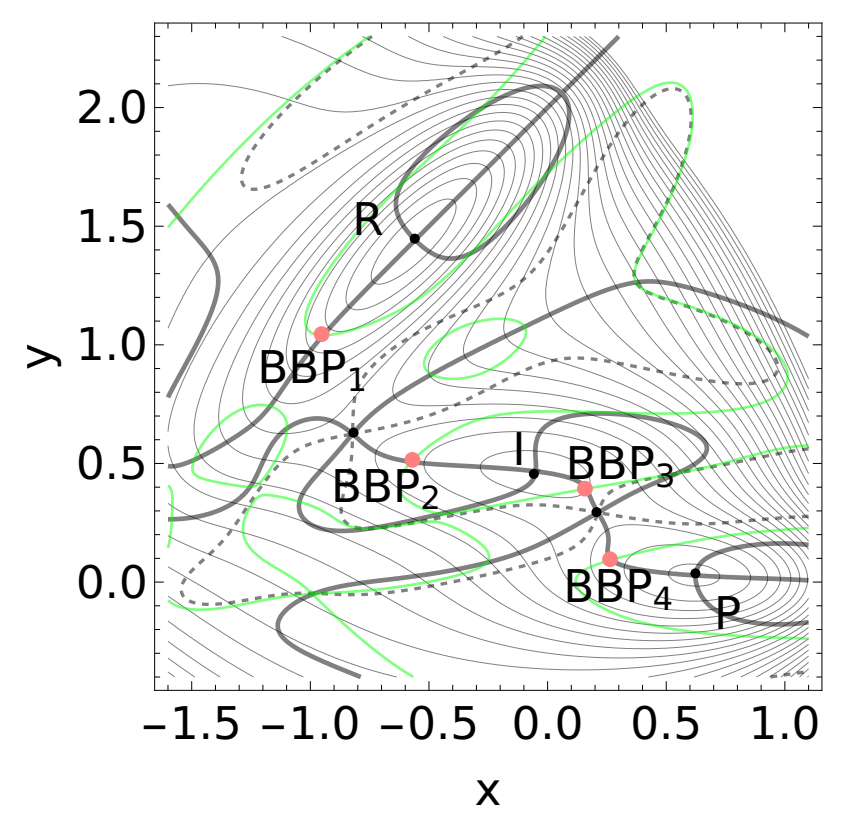

Figure 4: Surface of the MB function with GEs (gray), $\operatorname{Det}(H(x, y))=0$-lines (green), and convexity borders ${ }^{46}$ (dashes). Four optimal BBPs are red points.

They are near or at the 'minimum energy path'. Note that not all such crossings with a GE are chosen. Only the crossings near the stationary points, and in reaction valleys are 'optimal'. Other such crossings are calculated elsewhere. ${ }^{33}$ The dashed lines are the convexity border of the surface; they separate convex or concave level lines or equipotential curves. ${ }^{46}$

\section{Chemical force corridors on the MB surface}

The main direct chemical corridor for an enhanced pulling from minimum $R$ to $\mathrm{SP}_{1}$ is shown in Fig. 5, compare reference. ${ }^{19}$ After $\mathrm{SP}_{1}$ it further follows the full reaction valley from reactant, $R$, to intermediate, $I$, and product, $P$. The borders of the corridor are the singular NTs through the VRI points 1 and 4 . So we name it corridor $(1,4)$. The borders are drawn in blue and orange color. An optimal BBP is a red point (see Fig.4). VRIs are black points. Note that the 'optimal' $\mathrm{BBP}_{1}$ in the main valley of the global minimum is outside of this corridor to $\mathrm{SP}_{1}$. The reason is that the valley of the $\mathrm{SP}_{1}$ 'crosses' the main valley. The SPvalley is a side valley. (See a deeper discussion of this 'optimal' BBP below.) Three regular NTs of the corridor are shown by gray curves. They are representants of the corridor. ${ }^{47}$ They connect all stationary points along the reaction valley. Such a corridor is named type one. ${ }^{19}$ The regular NTs go in between the two colored border lines of the corridor. Note that they sometimes have turning points (TP) which are higher in energy than the neighboring SPs. At TPs the energy along an NT has a maximum. Remember that the corridor describes curves of FDSPs, but it does not directly describe pathways of chemical reactions.

Another direct corridor leads from minimum $R$ to $\mathrm{SP}_{2}$ through the mountains over $\mathrm{SP}_{1}$. It is shown in Fig. 6 . The corridor is very small. It avoids the crossing of $\mathrm{SP}_{1}$ and the intermediate minimum, $I$. In our classification of corridors ${ }^{19}$ such a case is still missing. The borders of the corridor are the singular NTs through the VRI points 1 and 2. We name it 


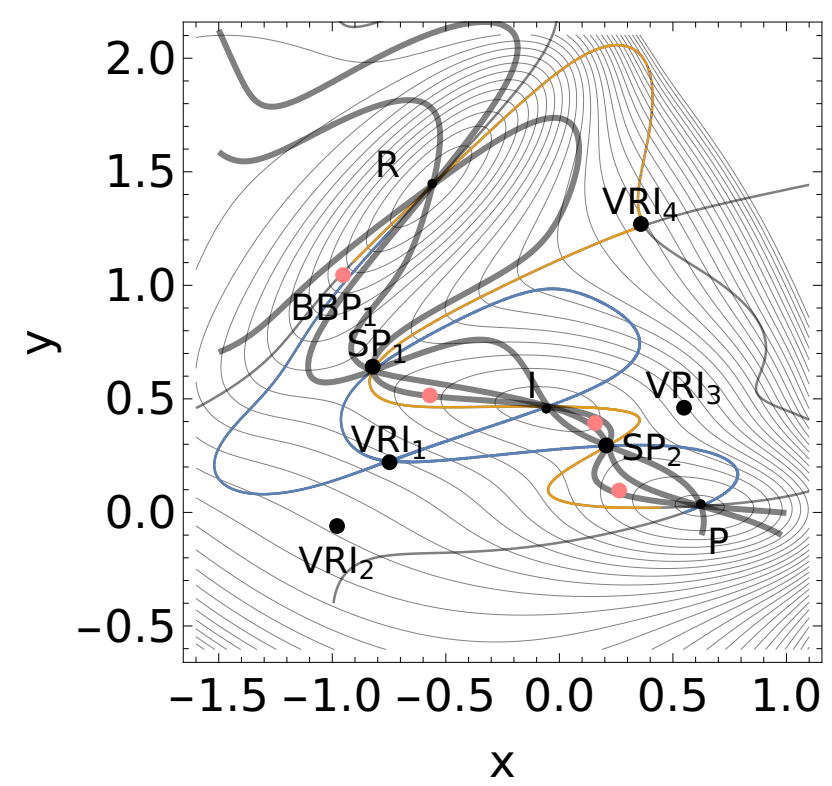

Figure 5: The main direct corridor from minimum $R$ to $\mathrm{SP}_{1}$ is also the reaction valley from $R$ to $P$. The borders of the corridor are the singular NTs through the VRI points 1 and 4. They are drawn in blue and orange color. An optimal BBP is a red point. VRIs are black bullets. Three regular NTs of the corridor are shown by gray curves. They connect all stationary points along the reaction valley.

corridor $(1,2)$. The borders are drawn in orange and blue color. Note that again the 'optimal' $\mathrm{BBP}_{1}$ is outside of this corridor. A regular NT of the corridor is shown by a gray curve. Here all the NTs of the corridor have TPs which are higher in energy than the neighboring SPs. We find a further specialty: yet, the full corridor crosses the $\mathrm{SP}_{2}$ not along the SP valley, but orthogonally to the valley direction, along the ridges of the SP.

Because the former corridor of Fig. 5 also crosses the $\mathrm{SP}_{2}$, we find here the strange case of a crossing of two direct corridors from global minimum, $R$, to the assumed product, $P$. We note again that in our classification of corridors ${ }^{19}$ such a case is missing. In contrast to model example 4.3 in ref. ${ }^{19}$ with three minimums and three SPs, and a maximum by an SP of index two in between, we miss here such an SP of index two between the two corridors. Here, they touch vice versa at the $\mathrm{VRI}_{1}$ and along the singular NT through $\mathrm{VRI}_{1}$.

Fig. 7 shows another type of circular corridors, ${ }^{19}$ named corridors of type 2. In panel (a) is shown the corridor $(2,3)$ between the border points $\mathrm{VRI}_{2}$ and $\mathrm{VRI}_{3}$. A representing $\mathrm{NT}$ of the corridor is drawn in bold gray. A force in such a direction can move together $\mathrm{SP}_{1}$ and $I$, and $\mathrm{SP}_{2}$ and $P$, see the next Section 5 for the corresponding effective surfaces. In Fig. $7 \mathrm{~b}$ is shown the half-corridor between the border points $\mathrm{VRI}_{3}$ and $\mathrm{VRI}_{4}$. It is named corridor $(3,4)$. It is likewise separated from the global minimum region around $R$. The other half corridor goes along the $R$-valley. Here $R$ moves forward or backward in its valley.

Further conceivable corridors, for example between the VRI points 1 and 3 , or between the VRI points 2 and 4, are combinations of the former corridors. The first $(1,3)$ is the combination of the corridors in Fig. 6 and Fig. 7 a, the second $(2,4)$ is the combination of Fig. 7, panels (a) and (b). 


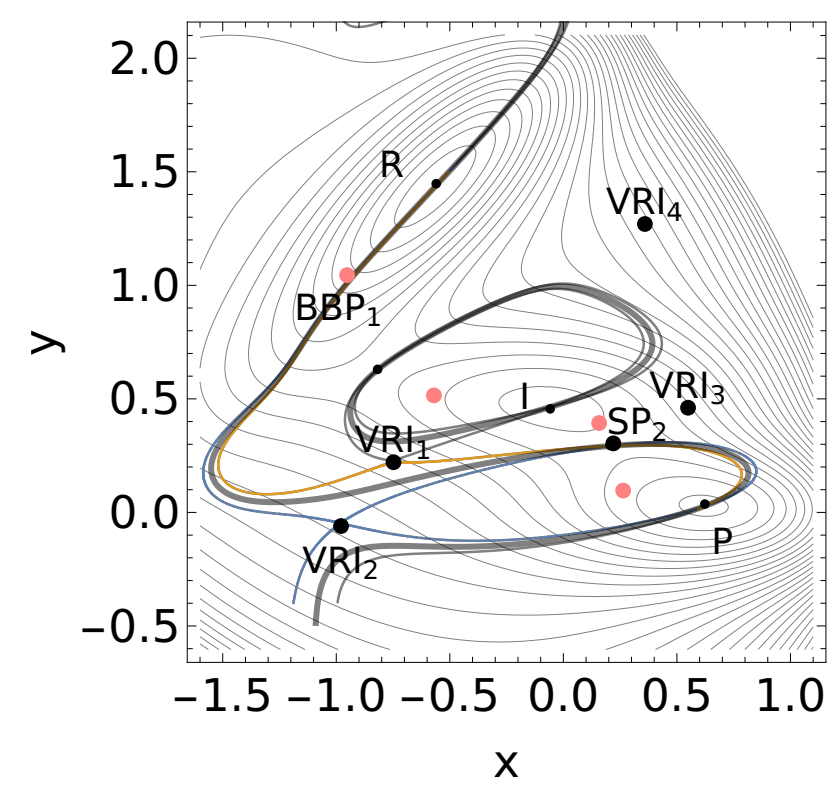

Figure 6: The direct corridor from minimum $R$ to $\mathrm{SP}_{2}$ and then to $P$. The borders of the corridor are the singular NTs through the VRI points 1 and 2. They are drawn in orange and blue color. A regular NT of the corridor is shown by a gray curve.

\section{Changes of effective surfaces under a force along the corridors on the MB surface}

\subsection{Main direct corridor}

In Fig. 8 one of the representative NTs along the minimum energy pathway from $R$ to $P$ is shown. It is in the direct corridor of type $1{ }^{19}$ compare with Fig. 5 . This NT crosses all further stationary points of the MB surface. In Fig. $8 \mathrm{a}$ we apply the force $\mathbf{f}=-55(0.4864,-0.8738)^{T}$. The effective surface is shown where a first 'catastrophe' happens: the intermediate minimum, $I$, and the $\mathrm{SP}_{2}$ have moved together, and here they coalesce to a shoulder, Sh. The intermediate is now over. In the interval around this force, $\mathbf{f}$, we would enforce a specific reaction from structures $I$ to $P$. The former minimums, $R$ and $P$, are only less changed. They keep their 'chemical meaning'.

At higher amounts of the force into the same direction, at $F=-285$ in MB units, the next panel (b) of Fig. 8 is drawn. Here the former global minimum, $R$, disappears in a shoulder with the former $\mathrm{SP}_{1}$. At this high amount of the force, the reaction from $R$ to $P$ is finished, at least.

The inverse direction of the force $\mathbf{l}=(0.4864,-0.8738)$ is shown in Fig. $8 \mathrm{c}$ with an amount of $F=124.87 \mathrm{MB}$ units. Under this force the $P_{\text {eff }}$ remains in a flat, moved minimum, however, the intermediate minimum and the former $\mathrm{SP}_{1}$ have coalesced. The point of their meeting is the shoulder, Sh, of the panel (c), exactly on the (green) BBP line. The intermediate again is over here.

Not shown is the next small increase of the amount $F$ to $128 \mathrm{MB}$ units. The effective surface under this force is similar to Fig. 8c, but now also the $\mathrm{SP}_{\text {eff }}$ and the $\mathrm{P}_{\text {eff }}$ of the last panel (c) coalesce and the back reaction $P$ to $R$ is fully enforced. 

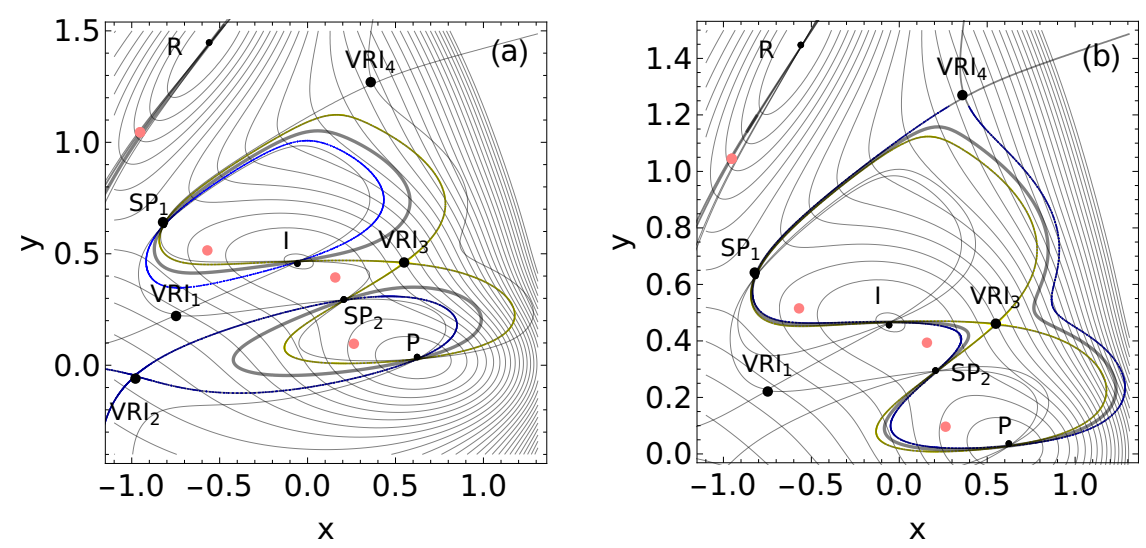

Figure 7: Two separated circular quarter-corridors in panel (a) between $\mathrm{SP}_{1}$ and minimum $P$, and a separated half-corridor in panel (b) for a reaction between $I$ and $P$. (The other half-corridor is the main valley of $R$.) The borders of the corridor are the singular NTs through the respective VRI points. They are drawn in orange and blue color. The not included other singular NTs are shown by thin curves. A representing regular NT of the respective corridor is shown by a bold gray curve.

\subsection{The second 'small' direct corridor}

The word 'small' in the headline concerns here the small region of possible directions for a force, in this corridor, see Fig.4. We will detect that the current effective surfaces can have broad reaction valleys. In Fig. 9 we treat the forward reaction for negative values of the force, $F=-89,-135$, and -185 in MB units. Fig. 9 a shows the emergence of a new shoulder at the 'Southern mountains', near point $(-1.1,0.1)$. It is a shoulder on the former ridge. The former global MEP of the MB surface can still be seen. In Fig. 9b the former global minimum has disappeared into a shoulder in a downward valley near point $(-1,1)$, and the shoulder of panel (a) has now developed into a new maximum in the center of the Figure, however, two further stationary points have emerged in the Figure below: a new SP and a new minimum. The new minimum below replaces the former global minimum of the MB surface. It may represent a quite new chemical structure of a corresponding molecule under the given force which does not exist without a load. Two different reaction pathways exist from $P_{\text {eff }}$ to the new minimum: one new way directly over the new SP, but also the old pathway from $P_{\text {eff }}$ over the old $S P_{2, \text { eff }}$ the old $I_{\text {eff }}$, and the old $S P_{1, \text { eff }}$. From $S P_{1, e f f}$ the new reaction path may circumvent the new maximum at the center. Note that $P_{\text {eff }}$ is still the deeper minimum on this effective surface in Fig. $9 \mathrm{~b}$.

In Fig. $9 \mathrm{c}$ the force is still more exited, and the former set of stationary points, $S P_{2, e f f}$, $I_{\text {eff }}$, and $S P_{1, \text { eff }}$ flatten out to two shoulders. The two minimums have now changed their order; the new minimum is now deeper.

Around $F=-225$ in MB units, at least, the former reactant, $P_{\text {eff }}$, and the new $S P_{\text {new }}$ coalesce and only the new minimum remains: this force makes a unite result. $R$ has disappeared, however, also $P$ is now missing. (This case is not shown in the Figure.)

The inverse direction of the force shows a totally different behavior of the effective surfaces, shown in Fig. 10. In panel (a) we show the movement of the former $S P_{1}$ 'uphill' on 

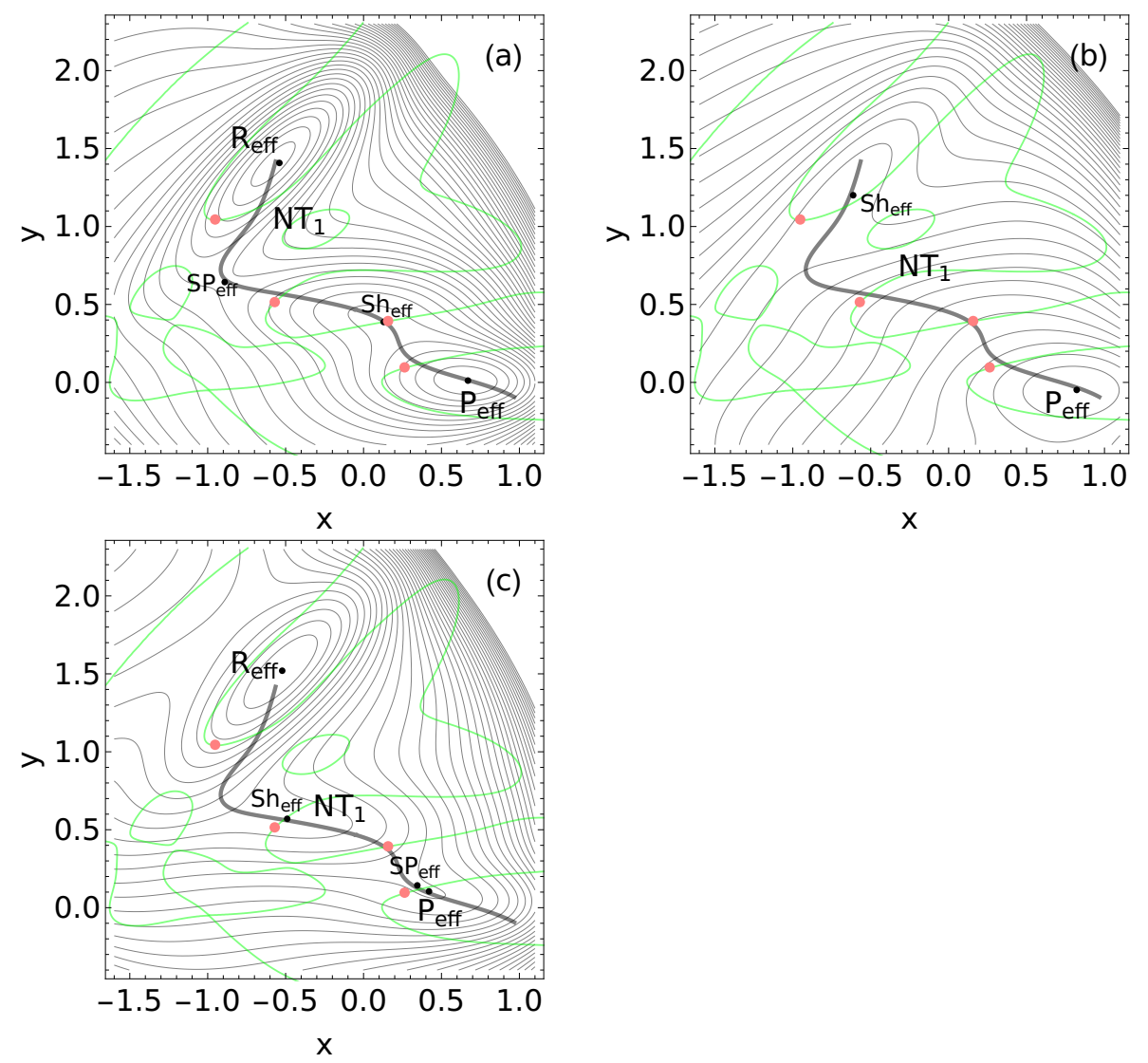

Figure 8: Effective surfaces under different amounts of force in direction of the given NT along the main corridor from minimum $R$ to $\mathrm{SP}_{1}$ to $I$, and to $\mathrm{SP}_{2}$ and to $P$, at least. The effective SPs and the effective minimums of the changed surfaces under the forces are all on the given NT which follows the selected direction $\mathbf{l}=(0.486,-0.874)^{T}$. Shown are the cases of $F=-55,-285$, and 124.9 in MB units. The forward reaction, panels (a,b), leaves $R$, the back reaction leaves $P$ in panel (c). The symbol 'Sh' means shoulder. Note that the green lines $\operatorname{Det}(\mathbf{H})=0$ are always equal on all effective surfaces. We repeat them for orientation.

the former ridge of the MB surface in direction to the $\mathrm{VRI}_{4}$. Of course, the SP moves on the NT which describes the direction of the force vector. The energy level of $P_{\text {eff }}$ is increased, in comparison to the former intermediate, $I$, and the barrier in between is decreased.

In Fig. 10b the former $S P_{1, \text { eff }}$ and the former $I_{\text {eff }}$ have moved together and form a shoulder. There remains a small barrier to the $P_{\text {eff }}$. Note that $R_{\text {eff }}$ has moved to the 'North-East' direction, on the NT, together with its deep global valley.

A further increase of the amount of force to $F=432$ in MB units, at least, will flatten out the effective $S P_{2, \text { eff }}$, and only the $R_{\text {eff }}$ remains. The former product valley has disappeared.

\subsection{Further Circular corridors}

We study the corridors between the VRI points 2 and 3, as well as between the VRI points 3 and 4. The corridors are shown in Fig.7. We take a regular NT from the corridors and develop a force in the corresponding direction. In both cases we face a situation where the 


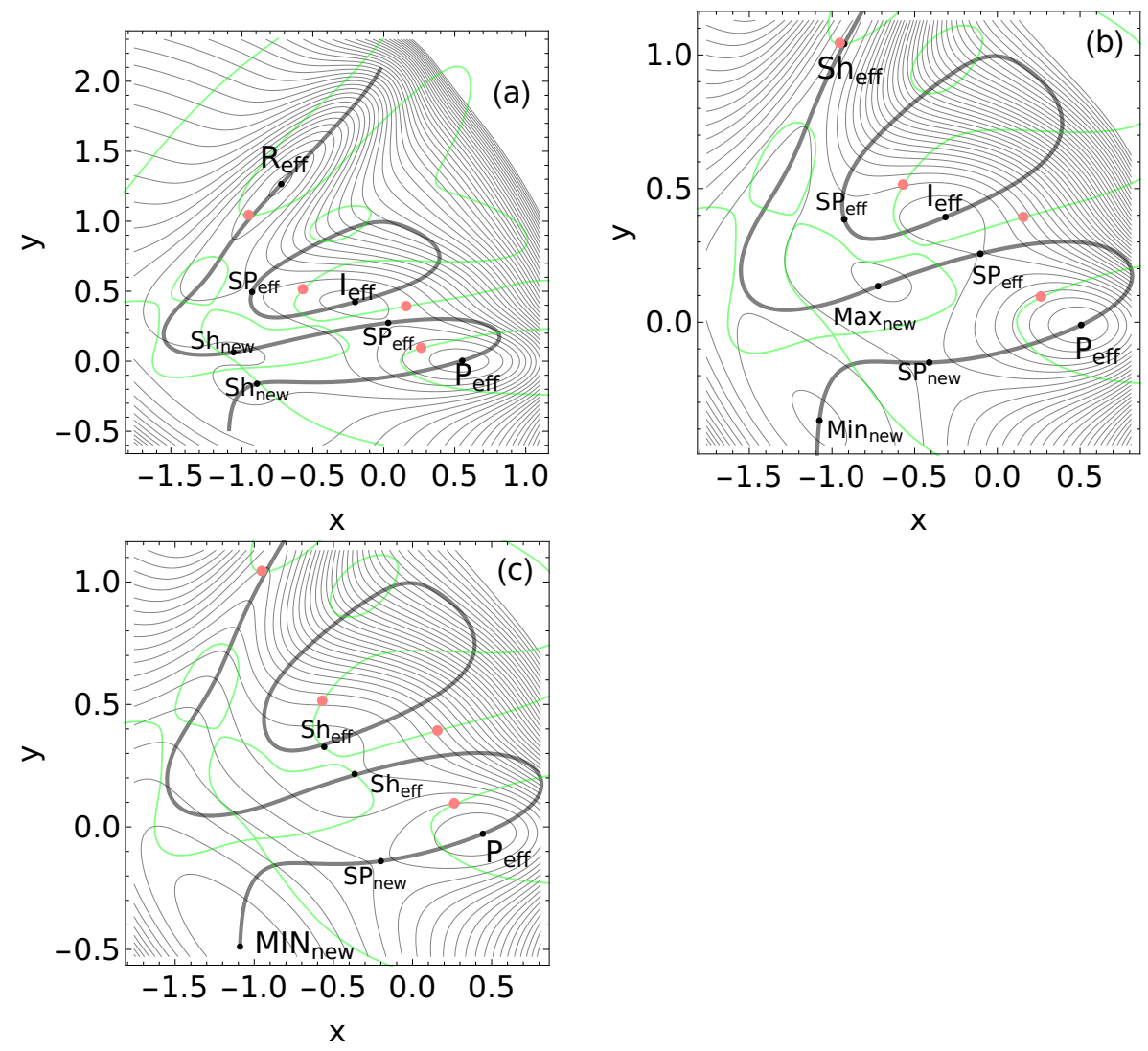

Figure 9: Effective surfaces under different amounts of force in direction of the NT along the second direct corridor from minimum $R$ to $\mathrm{SP}_{2}$, and to $P$, at least, compare with Fig. 6 . The effective SPs and the effective minimums of the changed surfaces under the forces are all on the one NT (thick gray) which follows the selected direction $\mathbf{l}=(-0.4296,-0.903)^{T}$. Shown are the three cases of $F=-89, F=-135$, and $F=-185$ in MB units. In all three panels one leaves $R$, by a forward reaction. The symbol 'Sh' means shoulder. Note that the green lines $\operatorname{Det}(\mathbf{H})=0$ are always the same, on all effective surfaces.

left branch of the NT does not cross any further stationary point of the MB surface: it is isolated. It cannot describe a reaction corridor from $R$ to another minimum.

In Fig.11 we use the direction $\mathbf{l}=(-0.619,-0.786)$ of an NT in the corridor $(2,3)$. The magnitude of the force is $F=309 \mathrm{MB}$ units in panel (a); but $F=-135 \mathrm{MB}$ units in panel (b) for the inverse direction of the given force.

In Fig.11a the effective reactant, $R_{e f f}$, moves with its bowl into 'North-East'-direction. The former $S P_{1}$ and the intermediate minimum have coalesced at a shoulder, $S h$, and the former product and its $S P_{2}$ have already moved close together.

For a quite stronger force, $F=545 \mathrm{MB}$ units, also the effective minimum, $P_{\text {eff }}$ and the former $\mathrm{SP}_{2}$ coalesce. Then only the moved reactant overlifes under the force, thus the back reaction is totally enforced. (This case is not shown.)

Fig.11 shoes the other direction of the same force. Here the effective reactant moves in its valley into the other direction. At the given force of $F=-135 \mathrm{MB}$ units it flattens out and disappears into a shoulder. A new minimum has emerged. The old $\mathrm{SP}_{1}$ and the old intermediate have coalesced to a shoulder. Only a flat minimum of $P_{\text {eff }}$ still exists. 

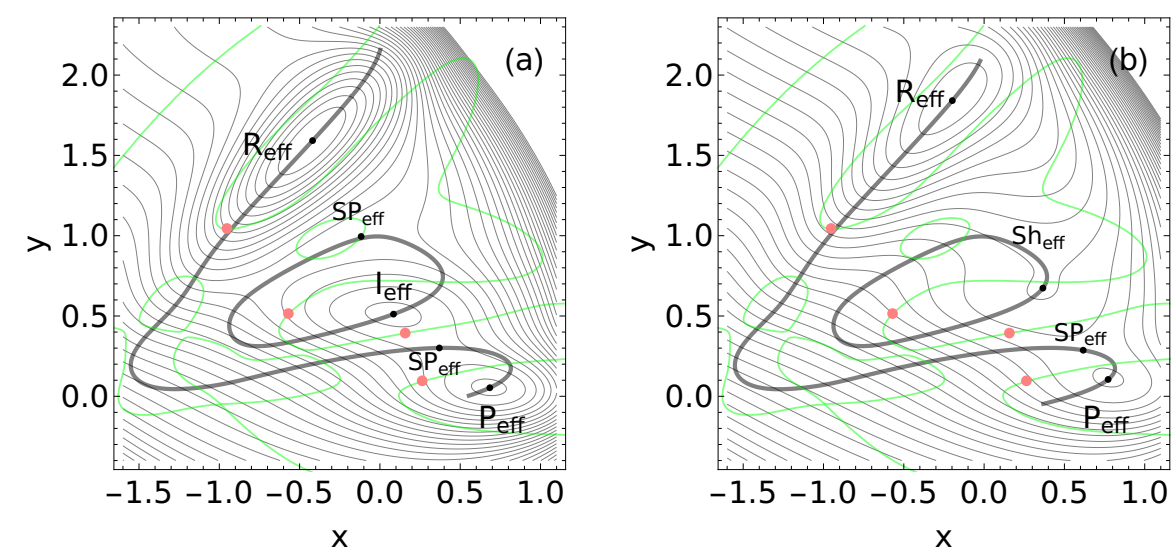

Figure 10: Effective surfaces under different amounts of force in the inverse direction of an NT along the second direct corridor from minimum $P$ to $R$, at least. The effective SPs and the effective minimums of the changed surfaces under the forces are all on the one $\mathrm{NT}_{2}$ which follows the selected direction $\mathbf{l}=(-0.4296,-0.903)^{T}$. Shown are the cases of $F=96$, and $F=288.5$ in $\mathrm{MB}$ units. In both panels one leaves $P$, by a backward reaction,

In both cases, the movement of the effective stationary points is 'constrained' to the two circular quarter branches of the NT of the force. One SP and one minimum move either on the 'left' arc, or on the 'right' arc of the corresponding circular branch, up to their coalescence.

It is not surprising that a new minimum can emerge. As it is possible that minimums or saddles can disappear under the force, the converse process is also possible. If the MB surface would be the model of a real molecular reaction, then under this force a new structure would appear which would not be possible without a load.

For a force of $F=-262 \mathrm{MB}$ units also the effective product minimum flattens out, and the back reaction to the new minimum is fully enforced. (This case is not shown.)

A reaction leaving the $R$ valley is not possible for this corridor; it only contains forbidden directions. The corresponding forces, forward or backward, enforce the back reaction from $P$ to $R$.

The next corridor concerns the family of NTs between the VRI points 3 and 4 . The corridor is shown in Fig.7b. A reaction leaving $R$ is again not possible, it is a forbidden direction. In Fig. 12 the special NT through the 'optimal' $\mathrm{BBP}_{1}$ point is shown. It belongs to the force $\mathbf{l}=(-0.809,-0.687)$. It is a representant of the current corridor. The NT is disconnected into two different branches. The left branch of the NT through $R$ is isolated. It cannot describe an enforced reaction corridor from $R$ to another minimum. No force of this direction can enforce that a reaction leaves the $R$ bowl. This is not possible, it is a forbidden direction. Now we discuss the effective surfaces under the force along the 'optimal' NT.

In Figs. $13 \mathrm{a}$ and $13 \mathrm{~b}$ we show the action of the force $\mathbf{l}=(-0.809,-0.687)$ which defines the $\mathrm{NT}_{\text {opt }}$ through $R$, on the MB surface. The different amounts $F=-80$, and $F=-155$ in MB units are used. The product, $P$, the $\mathrm{SP}_{2}$ and $\mathrm{SP}_{1}$, and the intermediate, $I$, move together and coalesce at different amounts of the force. First in Fig. 13a the $\mathrm{SP}_{1}$ and the intermediate, $I$, coalesce. A shoulder, Sh, is located on the second branch of the $\mathrm{NT}_{\text {opt }}$. Thus here $I$ 

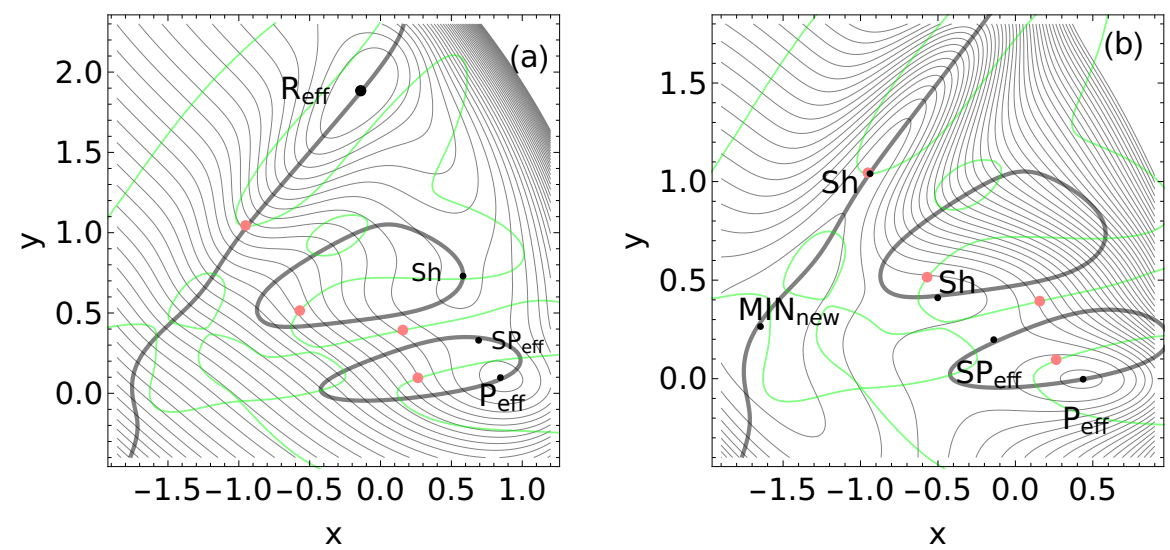

Figure 11: The effective surfaces under different amounts of force in direction of an NT in corridor $(2,3)$ along the circular corridors of Fig.7 (a). Shown are the cases of $F=309$ (a) and $F=-135$ in MB units (b). The effective SPs and the effective minimums of the changed surfaces under the force are all on the one NT which follows the selected direction.

disappears.

Later, for the nearly double amount of the force, in Fig. 13b, the former product, $P$, and the $\mathrm{SP}_{2}$ coalesce. Here the second 'catastrophe' emerges. Finally, the back reaction is only possible from $P$ to $R$. It is the one-way-case of the possible directions.

The reaction comes from the former product, $P$, downwards to the former reactant. However, this former reactant, $R_{e f f}$, has also disappeared. At $\mathrm{BBP}_{1}$ it is now an intermediate shoulder, Sh, which further points downwards to a new global minimum near point $(-1.9,0.3)$, named new $R_{e f f}$ in panel (b). If the MB surface describes a real chemical problem, then here a new structure emerges of the molecular system in question which does not exist without the outer force, $\mathbf{f}$.

The meaning of the point $\mathrm{BBP}_{1}$ is yet clear: it is the point where the structure of the former global minimum, $R$, disappears, while a new minimum emerges at another place on the $\mathrm{NT}_{\text {opt }}$, and a corresponding new $\mathrm{SP}$ can also emerge in between, in a small interval of the amount of the force.

Inversion of the chosen direction with $F=44$, and $F=675$ in MB units does not change the global reaction situation: we treat this in panels (c), and (d) of Fig. 13. First the intermediate, $I$, and the $\mathrm{SP}_{2}$ disappear in panel (c). Only $\mathrm{P}_{\text {eff }}$ remains below in the right corner. And in panel $(\mathrm{d})$ the situation shortly before the coalescence of $\mathrm{SP}_{2}$ and the former product $P$ is shown, under an exorbitant large amount of the force in MB units. Again it remains the only one reaction from $P$ to $R$. The location of $R_{\text {eff }}$ has now strongly moved to 'North-East' as well.

\section{Conclusion}

If we assume that the MB surface characterizes a reaction system then interesting conclusions can be found.

(i) There exist different corridors for possible movements of the stationary points (FDSPs) under a force: two direct corridors, one from $R$ over $I$ to $P$, or a neighboring corridor 


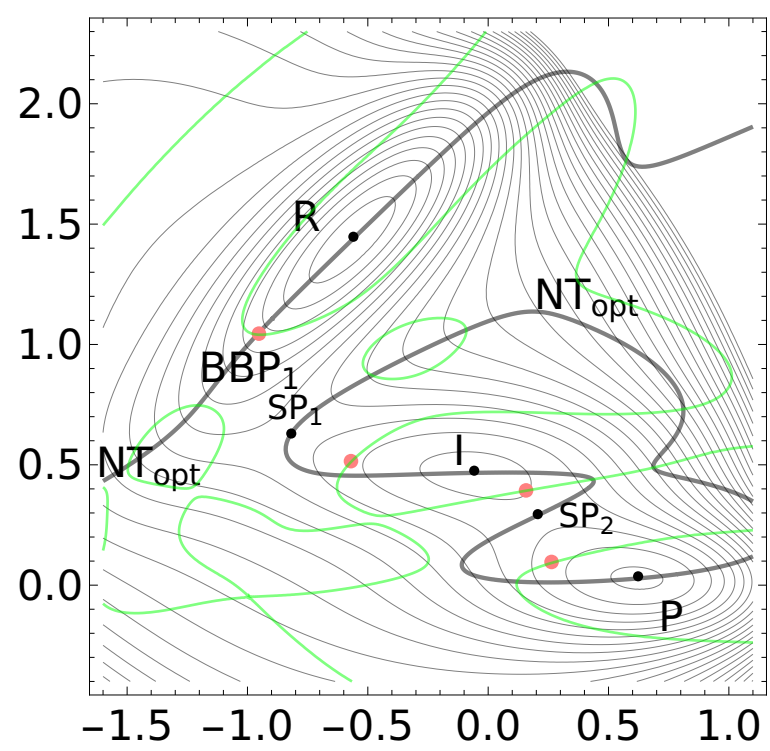

Figure 12: The 'optimal' NT along the main valley through the minimum $R$. The 'optimal' $\mathrm{BBP}_{1}$ is on this NT. However, this left branch of the NT does not cross any further stationary point of the MB surface: it is isolated. It cannot describe a force which drives the reaction from $R$ to another minimum, $I$ or $P$. A reaction leaving $R$ seems to be impossible, it is a forbidden direction. Optimal BBPs are red points.

from $R$ through the 'mountains' to $P$, and two separated corridors where circular NTs dictate the behavior.

(ii) On a separated branch of NTs along the main valley, near the $\mathrm{BBP}_{1}$, a new minimum emerges under strong forces which does not exist without a force. Note that $\mathrm{BBP}_{1}$ is not in the 'main'-corridors to $P$. On the other hand, separated circular branches of the leading NT indicate 'one-way'-properties of the enforced reaction. For example, no reaction $R \rightarrow P$ is possible, however, the back reaction is enforced for both force directions, forward or backward.

(iii) In comparison to our former classification of chemical corridors ${ }^{19}$ here exist two direct corridors from reactant, $R$, to product $P$, which cross at $\mathrm{SP}_{2}$.

Acknowledgments Financial support from the Spanish Ministerio de Economía y Competitividad, Project CTQ2016-76423-P is acknowledged.

\section{References}

1. Takacs, L. Chem Soc Rev 2013, 42, 7649.

2. Friscic, T.; James, S. L.; Boldyreva, E. V.; Bolm, C.; Jones, W.; Mack, J.; Steed, J. W.; Suslick, K. S. Chem Commun 2015, 51, 6248.

3. Makarov, D. E. J Chem Phys 2016, 144, 030901. 

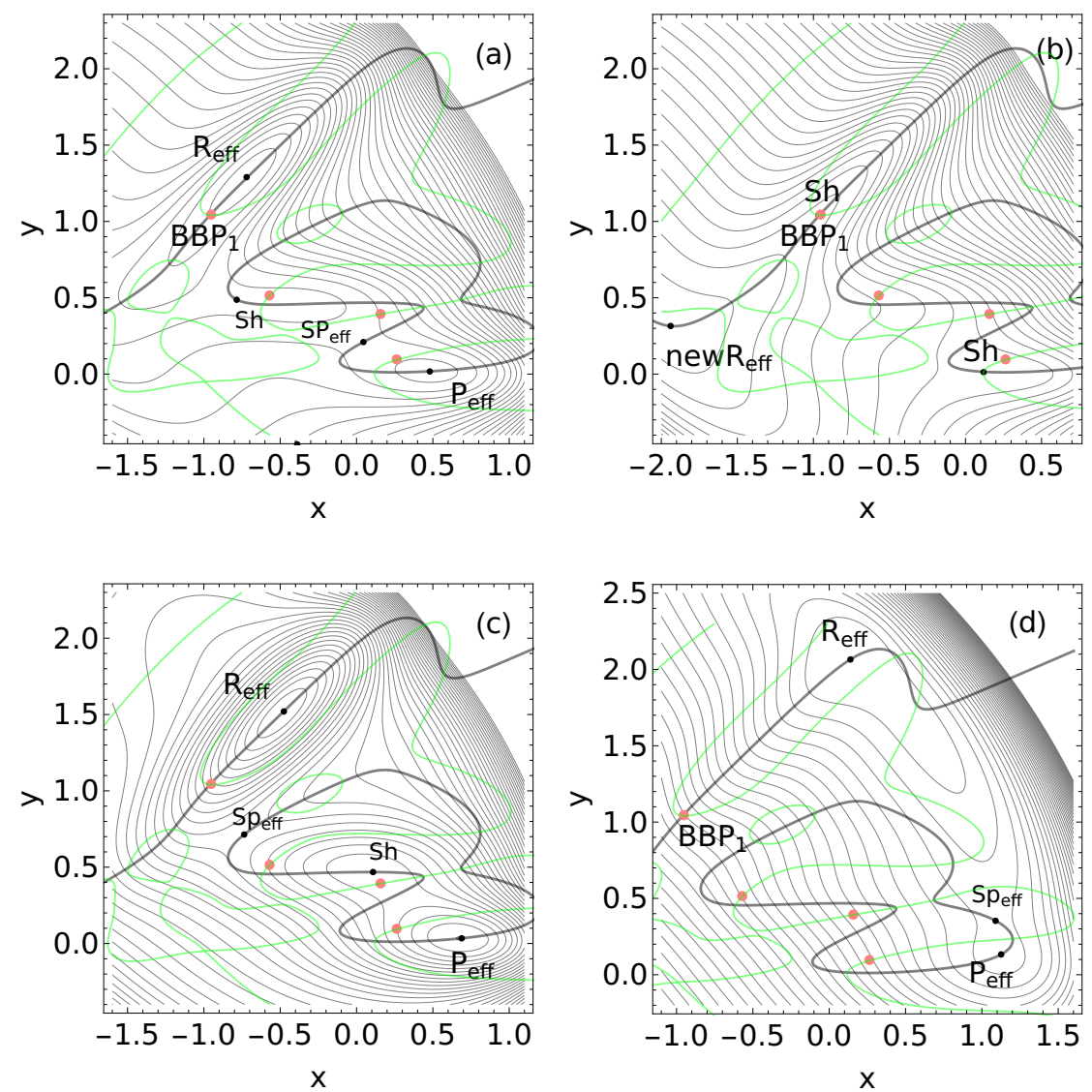

Figure 13: The effective surfaces under different amounts of force in direction of the 'optimal' $\mathrm{NT}_{\text {opt }}$ along the main valley through the minimum $R$. Shown are the cases of $F=-80,-155,44,675$ in MB units. A reaction leaving $R$ is not possible, it is a forbidden direction. The effective SPs and the effective minimums of the changed surfaces under the forces are all on the one $\mathrm{NT}_{\text {opt }}$ which follows the selected direction.

4. Thornton, E. R. J Am Chem Soc 1967, 89, 2915.

5. Eying, H.; Walter, J.; Rimball, G. E. Quantum Chemistry; John Wiley and Sons, Inc., New York, 1944.

6. Bell, G. I. Science 1978, 200, 618 .

7. Strunz, T.; Oroszlan, K.; Schumakovitch, I.; Güntherodt, H.-J.; Hegner, M. Biophys J 2000, 79, 1206 .

8. Bustamente, C.; Chemla, Y. R.; Forde, N. R.; Izhaky, D. Ann Rev Biochem 2004, 73, 705 .

9. Ribas-Ariño, J.; Shiga, M.; Marx, D. Angew Chem, Int Ed 2009, 48, 4190.

10. Wolinski, K.; Baker, J. Molec Phys 2009, 107, 2403.

11. Ribas-Ariño, J.; Marx, D. Chem Rev 2012, 112, 5412. 
12. Avdoshenko, S. M.; Makarov, D. E. J Phys Chem B 2015, 120, 1537.

13. Ong, M. T.; Leiding, J.; Tao, H.; Virshup, A. M.; Martínez, T. J. J Am Chem Soc 2009, 131,6377 .

14. Stauch, T.; Dreuw, A. Chem Rev 2016, 116, 14137.

15. Maeda, S.; Morokuma, K. J Chem Phys 2010, 132, 241102.

16. Maeda, S.; Ohno, K.; Morokuma, K. Phys Chem Chem Phys 2013, 15, 3683.

17. Sameera, W. M. C.; Sharma, A. K.; Maeda, S.; Morokuma, K. Chem Rec 2016, 16, 2349.

18. Quapp, W. J Math Chem 2004, 36, 365.

19. Quapp, W.; Bofill, J. M.; Ribas-Ariño, J. JPhysChemA 2017, 121, 2820.

20. Quapp, W.; Hirsch, M.; Imig, O.; Heidrich, D. J Comput Chem 1998, 19, 1087.

21. Quapp, W.; Hirsch, M.; Heidrich, D. Theor Chem Acc 1998, 100, 285.

22. Anglada, J. M.; Besalú, E.; Bofill, J. M.; Crehuet, R. J Comput Chem 2001, 22, 387.

23. Bofill, J. M.; Anglada, J. M. Theor Chem Acc 2001, 105, 463.

24. Crehuet, R.; Bofill, J. M.; Anglada, J. M. Theor Chem Acc 2002, 107, 130.

25. Quapp, W. J Theoret Comput Chem 2003, 2, 385.

26. Quapp, W. J Molec Struct 2004, 695-696, 95.

27. Bofill, J. M.; Quapp, W. J Chem Phys 2011, 134, 074101.

28. Quapp, W.; Bofill, J. M. Theoret Chem Acc 2016, 135, 113.

29. Quapp, W.; Bofill, J. M. J Comput Chem 2016, 37, 2467.

30. Branin, F. H. IBM J Res Develop 1972, 16, 504.

31. Quapp, W.; Bofill, J. M. J Phys Chem B 2016, 120, 2644.

32. Magnus, J. R.; Neudecker, H. Differential Calculus with Applications in Statistics and Econometrics; John Wiley \& Sons, Chichester, 1999.

33. Bofill, J.; Ribas-Ariño, J.; García, S.; Quapp, W. J Chem Phys 2017, 147, 152710.

34. Basilevsky, M.; Shamov, A. Chem Phys 1981, 60, 347.

35. Hoffmann, D. K.; Nord, R. S.; Ruedenberg, K. Theor Chim Acta 1986, 69, 265.

36. Quapp, W. Theoret Chim Acta 1989, 75, 447.

37. Schlegel, H. B. Theor Chim Acta 1992, 83, 15. 
38. Sun, J.-Q.; Ruedenberg, K. J Chem Phys 1993, 98, 9707.

39. Hirsch, M.; Quapp, W. Chem Phys Lett 2004, 395, 150.

40. Bofill, J. M.; Quapp, W.; Caballero, M. J Chem Theory Comput 2012, 8, 927.

41. Heidrich, D.; Kliesch, W.; Quapp, W. Properties of Chemically Interesting Potential Energy Surfaces; Springer: Berlin, Heidelberg, 1991.

42. Thom, R. Structural Stability and Morphogenesis: An Outline of a General Theory of Models; Addison-Wesley: Reading, MA, 1989.

43. Gilmore, R. Catastrophe Theory for Scientists and Engineers; Dover, New York, 1993.

44. Konda, S. S. M.; Avdoshenko, S. M.; Makarov, D. E. J Chem Phys 2014, 140, 104114.

45. Müller, K.; Brown, L. Theor Chim Acta 1979, 53, 75.

46. Hirsch, M.; Quapp, W. J Math Chem 2004, 36, 307.

47. Hirsch, M.; Quapp, W. J Molec Struct, THEOCHEM 2004, 683, 1. 